\title{
Perfil epidemiológico dos casos de sífilis congênita em um município de médio porte no nordeste brasileiro
}

\section{Epidemiological profile of cases of congenital syphilis a mid-sized municipality of Brazilian northeast}

\author{
Valdênia Cordeiro Lima ${ }^{1}$, Raquel Martins Mororó${ }^{1}$, Maria Aparecida Martins ${ }^{1}$, Sâmia Maria Ribeiro², Maria Socorro Carneiro \\ Linhares $^{2}$ \\ 1. Discente do curso de graduação em Enfermagem pela Universidade Estadual Vale do Acaraú (UVA), Sobral, Ceará, Brasil. 2. Docente do curso de graduação
} em Enfermagem pela Universidade Estadual Vale do Acaraú (UVA), Sobral, Ceará, Brasil.

\section{Resumo}

Introdução: A sífilis congênita é ocasionada pela transmissão vertical da bactéria, Treponema pallidum, ou seja, da gestante para o concepto por via transplacentária. É considerada como sendo um sério problema de saúde pública. Objetivo: Analisar o perfil epidemiológico dos casos de sífilis congênita no município de Sobral, Ceará. Metodologia: Levantamento epidemiológico, descritivo utilizando dados secundários com abordagem quantitativa. Os dados foram coletados a partir das notificações do Sistema de Informação de Agravos de Notificação, referentes ao período de 2008 a 2013. Foram analisadas variáveis sociodemográficas das gestantes, acompanhamento da gestação e relacionadas ao recém-nascido. Resultados: Foram diagnosticados e notificados 119 casos de sífilis congênita, sendo possível observar que a partir do ano de 2010 o número de casos aumentou substancialmente, passando de 9 casos para 45 em 2013. A incidência anual variou de 1,8 casos/1000 nascidos vivos, em 2008, a 13,8 casos/1000 nascidos vivos, em 2013. Quanto às características da assistência pré-natal, 115 gestantes realizaram pré-natal $(96,6 \%)$ e $74(62,1 \%)$ mulheres tiveram o diagnóstico da sífilis na gestação, sendo que apenas oito $(6,7 \%)$ tiveram o tratamento de forma adequada. Conclusão: 0 perfil da sífilis congênita do município estudado aponta para uma aceleração da incidência de casos no período analisado, embora dados mais recentes de 2015 apresentem redução significativa da notificação. A ocorrência dos casos da SC está amplamente relacionada com falhas no tratamento das gestantes infectadas, carecendo de preenchimento de lacunas no pré-natal que reforçam estratégias de prevenção dos casos.

Palavras-chave: : Análise de dados. Epidemiologia descritiva. Sífilis Congênita.

\begin{abstract}
Introduction: A Congenital syphilis is caused by the vertical transmission of the bacteria, Treponema pallidum, i.e., from the pregnant woman to the fetus through the placenta. It is considered as a serious public health problem. Objective: The analysis of the epidemiological profile of cases of congenital syphilis in the municipality of Sobral, Ceará. Methodology: Epidemiological survey, descriptive, using secondary data-exploratory study with a quantitative approach. The data were collected from the notification of the Information Service of failure in notification (Sistema de Informação de Agravos de notificação), for the period from 2008 to 2013. Several variables which included demographic data of the patients, monitoring of pregnancy related to the newborn were analyzed. Results: 119 cases of congenital syphilis, were diagnosed and notified making it is possible to observe that from the year 2010, the number of cases has increased substantially, from 9 to 45 cases in 2013. The annual incidence ranged from 1.8 cases per 1000 live births in 2008 , the 13.8 cases/1000 live births in 2013. As for the characteristics of prenatal care that 115 pregnant women received prenatal (96.6\%) and 74 (62.1\%) women had the diagnosis of syphilis in pregnancy, and only eight (6.7\%) had their treatment carried out in an appropriate manner. Conclusion: The profile of congenital syphilis of the city studied points to an acceleration of incidence of cases in the period analyzed, although more recent data from 2015 shows significant reduction of notification. The occurrence of cases of SC is largely related to failures in the treatment of infected pregnant women, lacking filling gaps in pre-natal which reinforce strategies for prevention of cases.
\end{abstract}

Key words: Analysis of data. Epidemiology. Syphilis Congenital.

INTRODUÇÃO

A sífilis é uma doença infeciosa causada pela bactéria Treponema pallidum, com transmissão predominantemente sexual. Nas gestantes, quando a sífilis não é tratada ou o esquema de tratamento é realizado de forma inadequada, a infecção é transmitida, por via transplacentária, ao concepto, ocasionando a sífilis congênita (SC). Essa doença pode ser classificada em precoce ou tardia, levando-se em consideração o surgimento da síndrome clínica, sendo respectivamente, antes e depois dos dois anos de vida. O diagnóstico para as duas formas é estabelecido por meio da associação de critérios epidemiológicos, clínicos e laboratoriais, podendo ser atentada a possibilidade de a criança ter sido exposta ao T. pallidum por via sexual ${ }^{1}$.

A SC tornou-se uma doença de notificação compulsória por meio da Portaria Ministerial № 542 de 22 de dezembro de $1986^{2}$. Já a sífilis em gestante somente passou a constar entre as doenças e agravos de notificação compulsória a partir de 2005, por meio 
da Portaria Ministerial № 33 de 14 de julho desse mesmo ano e a sífilis adquirida em 2011, com a Portaria Ministerial № 104, de 25 de janeiro ${ }^{4}$.

No Brasil, entre 1998 a junho de 2014, foram notificados no Sistema de Informação de Agravos de Notificação (SINAN) 104.853 casos de sífilis congênita em menores de 1 ano de idade. As regiões que apresentaram maiores registros de casos correspondem à Região Sudeste, com 48.015 casos (45,8\%), seguida da Região Nordeste com 32.884 (31,4\%). No Estado do Ceará de 2001 a 2013, foram notificados e investigados 5.953 casos de sífilis congênita em menores de 1 ano de idade ${ }^{5}$.

Em Sobral, município do Estado do Ceará, nos três últimos anos, observou-se um acentuado aumento em relação aos anos anteriores, com registro de 25 casos em 2011, 32 em 2012 e 45 em $2013^{6}$.

O Ministério da Saúde (MS), por meio do "Plano de Ação para Eliminação da Transmissão Vertical do HIV e da Sífilis Congênita", estabelecido pelos países da Organização Pan-Americana da Saúde (OPAS) em 2010, considerou como meta para 2015 a ocorrência de até 0,5 caso de sífilis congênita por 1.000 nascidos vivos. No Brasil, a ocorrência foi de 6,5 casos/1000 nascidos vivos, valor distante da meta aprovada para o referido ano ${ }^{7}$.

As ações de prevenção da SC estão estreitamente relacionadas aos cuidados com a gestante no pré-natal, como a realização dos testes sorológicos para sífilis no primeiro e terceiro trimestre de gestação, o tratamento correto e oportuno da infecção diagnosticada, instituído o mais precoce, tanto o da mulher quanto o do parceiro, no prazo máximo de finalização de até 30 dias antes do parto ${ }^{8}$.

Este trabalho investigativo integra as atividades do grupo tutorial do Programa de Educação pelo Trabalho para a Saúde, na temática Vigilância em Saúde, ora desenvolvido pela Universidade Estadual Vale do Acaraú, em parceria com a Secretaria Municipal de Saúde de Sobral e tem como objetivo analisar o perfil epidemiológico dos casos de sífilis congênita (SC) em um município de médio porte do nordeste brasileiro.

Espera-se contribuir para a reorganização das ações de controle da sífilis congênita integradas às ações dos profissionais de saúde na atenção básica e intervir na conjunção de fatores motivadores da ocorrência da doença.

\section{MÉTODOS}

Trata-se de um levantamento epidemiológico, descritivo, utilizando dados secundários com abordagem quantitativa. A utilização de documentos na construção de uma pesquisa deve ser valorizada e priorizada, pois a quantidade e a qualidade das informações que deles podemos extrair e resgatar revelam sua utilização em várias áreas de estudo?

O estudo foi realizado em Sobral, município localizado na região noroeste do Ceará, no Serviço de Vigilância Epidemiológica da Secretaria da Saúde do município. Participaram do estudo as crianças com sífilis congênita diagnosticadas e notificadas, residentes em Sobral, entre 1 de janeiro de 2008 e 31 de dezembro de 2013 e suas mães.

Os dados foram coletados em junho de 2015 a partir das fichas de notificação do SINAN, obtidas junto ao Serviço de Vigilância Epidemiológica da Secretaria de Saúde do Município de Sobral. No tocante aos dados sociodemográficos das gestantes, foram abordadas as seguintes variáveis: faixa etária, raça/ cor, escolaridade, ocupação e local de residência. As variáveis estudadas em relação ao acompanhamento da gestação foram: realização do pré-natal, momento do diagnóstico da sífilis na gestante, adequabilidade do tratamento e tratamento do parceiro concomitantemente à gestante. Quanto aos dados relacionados ao recém-nascido, as variáveis estudadas, foram: idade ao diagnóstico da SC, esquema de tratamento, presença de sinais e sintomas e evolução do caso.

O processamento e a análise dos dados foram feitos a partir dos softwares TabWin (DATASUS) e Excel (Microsoft ${ }^{\circledR}$ ), cujos resultados receberam tratamento simples da estatística descritiva e foram apresentados em forma de figuras e tabelas. O estudo incorporou os princípios da Resolução 466/2012 do Conselho Nacional de Saúde ${ }^{10}$. O projeto foi analisado e aprovado pelo Comitê de Ética em Pesquisa Envolvendo Seres Humanos da Universidade Estadual Vale do Acaraú (800.885/2014).

\section{RESULTADOS}

No período de 2008 a 2013 foram diagnosticados e notificados 119 casos de SC no município de Sobral, sendo possível observar que a partir do ano de 2010 o número de casos aumentou substancialmente, passando de nove para 45 casos em 2013. A taxa de incidência anual variou de 1,9 casos $/ 1000$ nascidos vivos, em 2008, a 13,8 casos/1000 nascidos vivos, em 2013 (Figura 1).

Figura 1. Número de casos notificados e incidência da sífilis congênita em Sobral- CE, 2008- 2013.

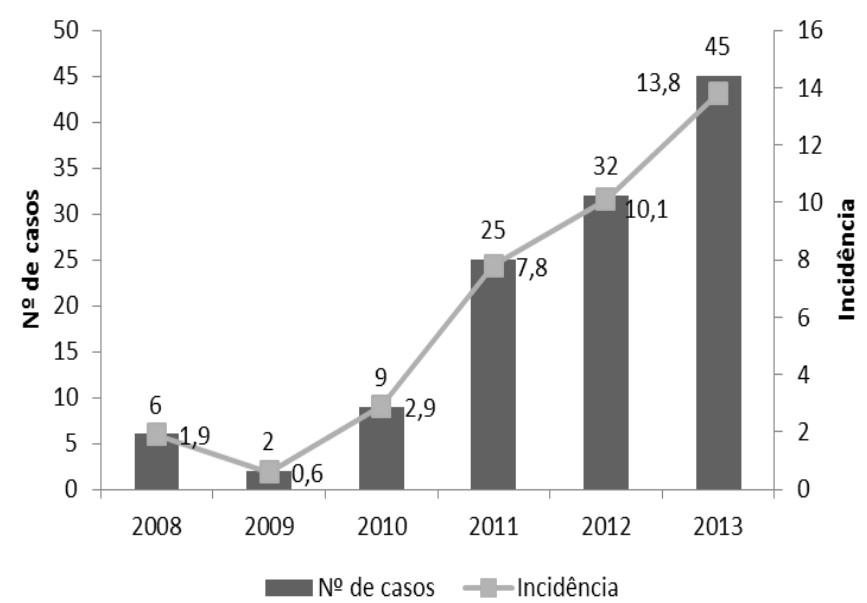


Em relação à variável idade materna, observa-se uma predominância na faixa etária de 20 a 25 anos, correspondendo a 53 casos, $44,5 \%$ do total de casos do período estudado, seguida por $22,7 \%$ na faixa de 26 a 30 anos, $18,5 \%$ com mais de 30 anos e em menor número as adolescentes, 15 a 19 anos, com $14,3 \%$ dos casos. A maioria das mulheres eram pardas $(92,4 \%)$, com ensino fundamental incompleto (43\%) e apenas uma $(0,8 \%)$ com ensino superior completo. Destaca-se que, em relação à escolaridade, uma elevada porcentagem desconhecia essa informação (30,3\%). A principal ocupação foi dona de casa $(72,2 \%)$ e eram residentes da zona urbana, $95 \%$ (Tabela 1 ).

Tabela 1. Distribuição das características maternas dos casos notificados de sífilis congênita em Sobral- CE, 2008- 2013.

\begin{tabular}{|c|c|c|}
\hline Características maternas & $\mathbf{N}$ & $\%$ \\
\hline \multicolumn{3}{|l|}{ Faixa etária (em anos) } \\
\hline 15 a 19 & 17 & 14,3 \\
\hline 20 a 25 & 53 & 44,5 \\
\hline 26 a 30 & 27 & 22,7 \\
\hline Mais de 30 & 22 & 18,5 \\
\hline \multicolumn{3}{|l|}{ Raça/cor } \\
\hline Parda & 110 & 92,4 \\
\hline Branca & 7 & 6,0 \\
\hline Amarela & 1 & 0,8 \\
\hline Preta & 1 & 0,8 \\
\hline \multicolumn{3}{|l|}{ Escolaridade } \\
\hline Sem escolaridade & 2 & 1,6 \\
\hline Fundamental Incompleto & 51 & 43 \\
\hline Fundamental Completo & 11 & 9,2 \\
\hline Médio Incompleto & 10 & 8,4 \\
\hline Médio Completo & 8 & 6,7 \\
\hline Superior Incompleto & 0 & 0 \\
\hline Superior Completo & 1 & 0,8 \\
\hline Ignorado & 36 & 30,3 \\
\hline \multicolumn{3}{|l|}{ Ocupação } \\
\hline Dona de casa & 86 & 72,2 \\
\hline Estudante & 5 & 4,2 \\
\hline Outras & 13 & 11 \\
\hline Ignorado & 15 & 12,6 \\
\hline \multicolumn{3}{|l|}{ Local de resodência } \\
\hline ZOna Ubana & 113 & 95 \\
\hline Zona Rural & 6 & 5 \\
\hline
\end{tabular}

Quanto às características da assistência pré-natal (Tabela 2), verifica-se que 115 gestantes realizaram pré-natal $(96,6 \%)$ e 74 $(62,1 \%)$ mulheres tiveram o diagnóstico da sífilis na gestação, sendo que apenas oito $(6,7 \%)$ realizaram o tratamento adequadamente. Entre as gestantes com o diagnóstico durante o pré-natal, menos da metade $(39,2 \%)$ finalizaram o tratamento pelo menos 30 dias antes do parto ressaltando-se que 2,7\% não o realizaram. Quanto ao parceiro sexual 95 (79,9\%) não o realizaram concomitantemente à gestante.

Tabela 2. Distribuição das características da assistência pré-natal das mães dos recém-nascidos notificados com sífilis congênita em Sobral- CE, 2008- 2013.

\begin{tabular}{lrr}
\hline Características da assistência pré-natal & N & \% \\
\hline Realizou pré-natal & & \\
\hline Sim & 415 & 96,6 \\
Não & & \\
\hline Diagnóstico da sífilis durante o pré-natal & 74 & 62,1 \\
\hline Sim & 45 & 37,8 \\
Não & & \\
\hline Tratamento das gestantes & 8 & 6,7 \\
\hline Adequado & 101 & 84,9 \\
Inadequado & 10 & 8,4 \\
\hline Não realizado & & \\
\hline
\end{tabular}

Tratamento da gestante finalizado pelo menos

30 dias antes do parto*

Sim $29 \quad 39,2$

$\begin{array}{lll}\text { Não } & 43 & 58,1\end{array}$

Tratamento não realizado

$2 \quad 2,7$

Tratamento do(s) parceiro(s) da(s) gestantes

Sim $16 \quad 13,4$

$\begin{array}{lll}\text { Não } & 95 & 79,9\end{array}$

$\begin{array}{lll}\text { Ignorado } & 8 & 6,7\end{array}$

*Entre as mulheres que tiveram o diagnóstico de sífilis durante o prénatal.

Com relação à variável idade ao diagnóstico da SC, a maioria $(88,2 \%)$ se encontrava com até dois dias de vida. Em 78,2 \% dos casos, o tratamento realizado foi com Penicilina $G$ Cristalina 100.000 a $150.00 \mathrm{UI} / \mathrm{kg} / \mathrm{dia}, 93,3 \%$ não apresentaram sinais e sintomas da doença e em $94 \%$ houve evolução com vida.

\section{DISCUSSÃO}

O aumento do número de casos de SC notificados ao longo da série histórica estudada pode ser atribuído a diversos fatores, como diminuição do número de sub-registros e consequentemente o aumento das notificações, bem como ações de vigilância epidemiológica direcionadas para uma melhor abordagem e identificação dos casos da doença no município.

O MS estabeleceu junto a OPAS como meta a redução da SC para menos de 0,5 caso/1000 nascidos vivos até o ano de 2015, para que a doença seja considerada eliminada como problema de saúde pública ${ }^{7}$ Considerando o parâmetro de eliminação 
estabelecido para 2015, o município de Sobral embora com uma redução da incidência comparada com a encontrada em 2013, último ano de avaliação do presente estudo (13,8 casos/1000 nascidos vivos), ainda está distante de atingir sua meta. Em 2015, foram notificados 19 casos, alcançando uma taxa de incidência de 5,5 casos/1000 nascidos vivos ${ }^{6}$.

Elevadas taxas de ocorrência da SC são, também, encontradas em diferentes regiões do País. Em estudo semelhante realizado em Natal - RN, no período de 2004 a 2007, encontrou-se uma incidência média de SC de 6 casos/1000 nascidos vivos, sendo maior no ano de $2006 \mathrm{com}$ a taxa de 7,1/1000 nascidos vivos ${ }^{11}$. A taxa de incidência de 6 casos $/ 1000$ nascidos vivos também foi encontrada no município do Rio de Janeiro, em um estudo que analisou os casos de SC notificados nos anos de 2007 e $2008^{12}$.

No que se refere às variáveis maternas, encontrou-se uma incidência de crianças com SC, cujas mães se encontravam na faixa etária de 20 a 25 anos, seguida pela de 26 a 30 anos, mostrando que essas mulheres se encontram na fase adulto jovem. No Distrito Federal, as mães com idade entre 19 a 35 anos, representaram $53,7 \%$ da amostra analisada ${ }^{13}$.

Com relação à raça/cor e à escolaridade materna, este estudo evidenciou resultados semelhantes aos encontrados em estudos realizados na Bahia ${ }^{14}$ e em Minas Gerais ${ }^{15}$ cuja maior predominância foi entre mães pardas e com ensino fundamental incompleto. Ressalta-se o elevado número de fichas com o campo da escolaridade em branco ou preenchido como ignorado, o que compromete uma análise fidedigna dessa informação.

A principal ocupação identificada das mães das crianças com SC foi dona de casa; porém, sabe-se que esse campo da ficha de notificação rotineiramente é ignorado ou é preenchido de maneira inadequada pelo profissional que realiza a notificação. Estudo realizado com mulheres em Pernambuco, sobre vulnerabilidade para a sífilis, identificou que apenas $25,5 \%$ das mulheres entrevistadas referiam exercer trabalho remunerado ${ }^{16}$.

No que diz respeito às características da assistência pré-natal, evidenciou-se que existe uma boa adesão das mulheres a essas consultas, mostrando que a quase totalidade dos casos em estudo frequentavam essa modalidade de atendimento na gestação. Isso representa um momento de grande valia para a mulher, para fins de diagnóstico, tratamento, promoção da saúde e prevenção de possíveis intercorrências oriundas do período gestacional, inclusive previne problemas relacionados à saúde do concepto ${ }^{17}$.

Durante o pré-natal, o MS preconiza a realização de dois Venereal Disease Research Laboratory (VDRL), sendo um no primeiro e outro no terceiro trimestre de gestação, além daquele realizado na entrada à maternidade, no momento do parto ${ }^{1}$. Assim, para a prevenção da sífilis congênita, é oportuno se realizar no pré-natal, o mais precocemente possível, o diagnóstico e o tratamento da sífilis na gestante e de seu parceiro sexual ${ }^{12}$.
Para ser considerado como adequado o tratamento da sífilis na gestante, o MS estabeleceu alguns critérios a serem contemplados, sendo eles: tratamento com penicilina benzatina, a ser realizado por completo com aplicação das doses do esquema terapêutico adequado à fase clínica da doença, primária, secundária ou latente, parceiro sexual tratado concomitantemente à gestante e tendo sido finalizado pelo menos 30 dias antes do parto ${ }^{1}$. No presente estudo, apenas oito gestantes preencheram esses critérios e tiveram seu tratamento considerado como adequado.

Apesar do resultado eficiente de adesão das mulheres às consultas de pré-natal $(96,6 \%)$, foi possível observar que quase $40 \%$ das gestantes tiveram o diagnóstico no momento do parto e que, entre as que tiveram o diagnóstico durante o prénatal, menos da metade completou o tratamento em menos de 30 dias antes do parto. Estas situações comprometeram o tratamento oportuno da sífilis nas gestantes e podem ter levado ao aumento de casos da SC. Muitos fatores podem relacionarse à falta de tratamento oportuno da gestante, como a demora no diagnóstico, a falta do medicamento para o tratamento, o desconhecimento da magnitude do agravo ou a resistência das pacientes ao tratamento ${ }^{12,18,19}$.

Sabe-se que a sífilis é uma doença que acomete parcelas menos favorecidas socialmente em que os fatores sociais são mais acentuados. Esse fator pode corroborar uma deficiência em seu autocuidado e comprometimento na realização do tratamento ${ }^{18}$.

A partir dos resultados, observa-se que, além de outros fatores, a falta do tratamento do parceiro sexual da maioria das gestantes com sífilis, pode ter contribuído muito para as possibilidades de reinfecção e falhas na adequabilidade do tratamento das mulheres.

Situação semelhante à observada em Sobral foi encontrada em um estudo realizado em Roraima, em que nenhum dos parceiros das parturientes analisadas realizou o tratamento ${ }^{20}$. A abordagem para a inclusão de parceiros sexuais nos tratamento de doenças pode não ser uma tarefa fácil para a equipe de profissionais de saúde. Muitas vezes, a falta de adesão dos parceiros sexuais aos tratamentos de sífilis está relacionada à falta ou à baixa capacidade do profissional para identificar e/ ou conduzir um plano terapêutico mais adequado a cada caso. Fazse necessário criar um vínculo com esses parceiros, a fim de que eles possam compreender sua responsabilidade e importância no resultado do tratamento.

No que concerne às características dos casos de sífilis congênita, conclui-se que, apesar dos diagnósticos da sífilis em gestantes não terem sido realizados em tempo oportuno e tratamentos de maneiras adequadas, sejam pela falta da adesão dos parceiros sexuais ou falhas na aplicação de todas as doses de penicilina benzatina, o mesmo não aconteceu com as crianças que foram infectadas. O diagnóstico foi realizado logo após o nascimento e todos receberam tratamentos, conforme o esquema padronizado. 
Evidenciou-se que, na totalidade dos casos, nascem crianças sem sintomatologia para sífilis congênita. Esse fato merece uma investigação, principalmente pela equipe de profissionais da maternidade e da atenção básica, onde vão realizar o acompanhamento da criança. Segundo o MS, o exame de VDRL pode positivar com poucos meses após o nascimento ${ }^{1}$.

As ações de saúde desenvolvidas durante a atenção ao prénatal devem dar cobertura a toda a população de gestantes, assegurando o acompanhamento, a continuidade no atendimento e a avaliação ${ }^{21}$, como diagnóstico e tratamento precoce da sífilis adquirida 22 .

A partir dessa análise, entendemos que se faz necessário melhorar as ações para prevenção da sífilis congênita nos serviços de saúde da atenção básica, focalizando na deteç̧ão precoce da gestante, no diagnóstico e tratamento oportuno da sífilis adquirida, assim como na captação dos parceiros. Esses devem receber uma abordagem desde o início da gestação de sua parceira, sendo sensibilizados quanto aos riscos que a sífilis poderá acarretar à criança acometida por essa infecção.

No que concerne às características maternas, os dados analisados refletem que a sífilis ainda é uma doença que acomete em especial aos menos favorecidos socialmente, com predomínio de mulheres com pouco estudo e tendo como ocupação ser dona de casa. Além disso, verifica-se um destaque maior dos casos na faixa de adultos jovens, evidenciando que as ações educativas devem ter um enfoque maior para esse grupo tendo em vista sua maior vulnerabilidade.

Quanto às características inerentes ao pré-natal das mães que tiveram filhos com SC, entendemos que há uma boa cobertura de pré-natal ofertada. No entanto, existe ainda uma dificuldade em relação ao tratamento, sendo este realizado de maneira inadequada tanto pela mulher quanto pelo parceiro sexual, na maioria dos casos analisados.

Corroborando esses dados, entendemos que é necessário maior empenho por parte dos profissionais para lidar com esses casos, por meio da busca ativa, e também trabalhar com uma abordagem que aproxime e sensibilize os pais em relação a esse tratamento, fazendo que se sintam responsáveis pela saúde e vida daquele concepto.

O perfil da sífilis congênita do município estudado aponta para uma aceleração da incidência de casos no período analisado, embora dados mais recentes de 2015 apresentem redução significativa da notificação. A ocorrência dos casos da SC está amplamente relacionada com falhas no tratamento das gestantes infectadas, carecendo de preenchimento de lacunas no pré-natal que reforcem as estratégias de prevenção dos casos.

Destaca-se a limitação do presente estudo ao utilizar fontes de dados secundários, do SINAN, com prováveis sub-registros e/ou subnotificações de casos.

\section{REFERÊNCIAS}

1. Ministério da Saúde [BR], Secretaria de Vigilância em Saúde, Programa Nacional de DST/AIDS, Diretrizes para controle da sífilis congênita: manual de bolso. 2. ed. Brasília: Ministério da Saúde; 2006.

2. Brasil. Ministério da Saúde. Portaria no 542, 22 de dezembro de 1986. Dispõe sobre o Sistema Nacional de Vigilância Epidemiológica e dá outras providências. Diário Oficial [da] República Federativa do Brasil. 1986 Dez 24; Seção 1. p. 19827.

3. Brasil. Ministério da Saúde. Portaria no 33, de 14 de julho de 2005. Inclui doenças à relação de notificação compulsória, define agravos de notificação imediata e a relação dos resultados laboratoriais que devem ser notificados pelos Laboratórios de Referência Nacional ou Regional. Diário Oficial [da] República Federativa do Brasil, 2005 Jul 14; Seção I. p. 111.

4. Brasil. Ministério da Saúde. Portaria no portaria 104, de 25 de janeiro de 2011. Define as terminologias adotadas em legislação nacional, conforme o disposto no Regulamento Sanitário Internacional 2005 (RSI 2005), a relação de doenças, agravos e eventos em saúde pública de notificação compulsória em todo o território nacional e estabelece fluxo, critérios, responsabilidades e atribuições aos profissionais e serviços de saúde. Diário Oficial [da] República Federativa do Brasil. 2011 Jan 26; Seção 1. p. 37.

5. Ministério da Saúde [BR]. Secretaria de Vigilância em Saúde. Departamento Nacional de DST, AIDS e Hepatites Virais. Boletim epidemiológico: Sífilis. Brasília [internet]. 2015 [acesso 2016 Abril 20]. Disponível em: http://www.aids.gov.br/ publicacao/2015/boletim-epidemiologico-de-sifilis-2015

6. Ministério da Saúde [BR]. Secretaria de Vigilância em Saúde. Departamento de DST/AIDS e Hepatites Virais. Indicadores e dados básicos da sífilis nos municípios brasileiros. Brasília: Ministério da Saúde; 2016 [acesso 2016 Maio 2]
Disponível em: http://indicadoressifilis.aids.gov.br/

7. Ministério da Saúde [BR]. Transmissão vertical do HIV e Sífilis: estratégias para a redução e eliminação. Brasília: Ministério da Saúde; 2014. 18p.

8. São Paulo. Secretaria de Estado da Saúde. Centro de controle de doenças. Programa Estadual de DST/Aids. Centro de Referência e Treinamento DST/ Aids. Guia de Bolso para o manejo da sífilis em gestante e sífilis congênita. São Paulo: Secretaria de Estado da Saúde. 2016. A versão de 2016 não foi localizada. Disponível em: http://www.saude.campinas.sp.gov.br/doencas/ sifilis/guiadebolsodasifilis_2edicao2016.pdf

9. Silva JRS, Almeida CD, Guindan JF. Pesquisa documental: pistas teóricas e metodológicas. Revista Brasileira de História \& Ciências Sociais. 2009 Jul [acesso 2015 Dez 18]; 1(1): 1-15.

10. Brasil. Ministério da Saúde. Conselho Nacional de Saúde. Resolução № 466/2012. Aprova diretrizes e normas regulamentadoras de pesquisa envolvendo seres humanos. Diário Oficial [da] República Federativa do Brasil. 2013 Jun 13. Seção 1. p. 59

11. Holanda MTCG, Barreto MA, Machado KMM, Pereira RC. Perfil epidemiológico da sífilis congênita no Município do Natal, Rio Grande do Norte - 2004 a 2007. Epidemiol. Serv. Saúde [Internet]. 2011 Jun [acesso 2015 Dez 17]; 20( 2 ): 203-212. Disponível em: http://scielo.iec.pa.gov.br/scielo. php?script=sci_arttext\&pid=S1679-49742011000200009.

12. Domingues RMSM, Saracen V, Hartz ZMA, Leal MC. Sífilis congênita: evento sentinela da qualidade da assistência pré-natal. Rev. Saúde Pública [Internet]. 2013 Fev [acesso 2015 Dec 16]; 47(1): 147-157. Disponível em: http://www. 
scielo.br/scielo.php?script=sci_arttext\&pid=S0034-89102013000100019

13. Magalhães DMS, Kawaguchi IAL, Dias A, Calderon IMP. Sífilis materna e congênita: ainda um desafio. Cad. Saúde Pública [Internet]. 2013 Jun [acesso 2016 Abril 17]; 29(6): 1109-1120.

14. Oliveira JS, Santos JV. Perfil epidemiológico da sífilis congênita no estado da Bahia, no período de 2010 a 2013. Rev. Eletrôn. Atualiza Saúde. 2015 jul./dez; 2(2): 20-30.

15. Lima MG, Santos RFR, Barbosa GJA, Ribeiro GS. Incidência e fatores de risco para sífilis congênita em Belo Horizonte, Minas Gerais, 2001-2008. Ciênc. Saúde Coletiva [Internet]. 2013 Feb [citado 2016 Abril 27];18(2): 499-506. Disponível em: http://www.scielo.br/scielo.php?pid=S1413$81232013000200021 \&$ script=sci_abstract\&tlng=pt.

16. Henriques MFCM. Vulnerabilidade para sífilis congênita: estudo descritivo em maternidades com Projeto Nascer em Pernambuco [dissertação]. Recife (PE): Instituto Materno-Infantil Professor Fernando Figueira; 2008.

17. Ministério da Saúde [BR]. Secretaria de Atenção à Saúde. Departamento de Atenção Básica. Atenção ao pré-natal de baixo. Brasília: Ministério da Saúde; 2013.

18. Hildebrand VLPC. Sífilis congênita: fatores associadas ao tratamento das gestantes e seus parceiros [dissertação]. Rio de Janeiro (RJ): Escola Nacional de Saúde Pública Sérgio Arouca; 2010.

19. Araújo CL, Shimizu HE, Sousa AIA, Hamann EM. Incidência da sífilis congênita no Brasil e sua relação com a Estratégia Saúde da Família. Rev. Saúde Pública [Internet]. 2012 Jun [acesso 2016 Maio 10]; 46(3): 479-486. Disponível em: http:// www.scielo.br/scielo.php?script=sci_arttext\&pid=S0034-89102012000300010.

20. Lins CDM. Epidemiologia da sífilis gestacional e congênita no extremo setentrional da Amazônia [dissertação]. Boa Vista (RR): Universidade Federal de Roraima; 2014.

21. Ximenes FRG Neto, Leite JL, Fuly PSC, Cunha ICKO, Clemente AS, Dias MSA et al . Qualidade da atenção ao pré-natal na Estratégia Saúde da Família em Sobral, Ceará. Rev. bras. Enferm . [Internet]. 2008 Oct [aceso 2016 Maio 05]; 61(5): 595-602. Disponível em; http://www.scielo.br/scielo.php?script=sci_ arttext\&pid=S0034-71672008000500011.

22. Clemente TS, Lima MM, Barros LA, França AMB, Bento TMA. A importância do pré-natal como ferramenta na prevenção da sífilis congênita: revisão bibliográfica. Cadernos de Graduação - Ciências Biológicas e da Saúde Fits [Internet]. 2012 Nov [acesso 2016 Maio 05];1(1): 33-42. Disponível em: https:// periodicos.set.edu.br/index.php/fitsbiosaude/article/view/455.

\section{Como citar este artigo/How to cite this article:}

Lima VC, Mororó RM, Martins MA, Ribeiro SM, Linhares MSC. Perfil epidemiológico dos casos de sífilis congênita em um município de médio porte no nordeste brasileiro. J Health Biol Sci. 2017 Jan-Mar; 5(1):56-61. 